\title{
Polyethylene Liner Dissociation with the Depuy Pinnacle Cup: A Report of 6 Cases
}

\author{
Neal Singleton* \\ Department of Orthopaedic, Whakatane Hospital, New Zealand \\ *Corresponding author: Neal Singleton, Department of Orthopaedic, Whakatane Hospital, Whakatane, New Zealand

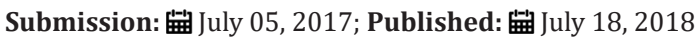

\begin{abstract}
This case report discusses six cases of liner dissociation with the Pinnacle acetabulum following primary total hip arthroplasty. Cases involved a $54 \mathrm{~mm}, 55 \mathrm{~mm}, 56 \mathrm{~mm}$ or $58 \mathrm{~mm}$ Pinnacle cup with a neutral polyethylene liner and a $28 \mathrm{~mm}$ femoral head with either an uncemented Corail or cemented C-Stem femoral prosthesis. Liner dissociation occurred between six months and 19 months post-operatively and all cases occurred following hip flexion. Revision involved replacement of the dissociated liners with either a neutral or lipped liner (Pinnacle or Ultamet polyethylene or metal) and in four of the six cases the femoral head size was increased. In all six cases the dissociated liners were found to have three of the six peripheral locking tabs sheared off. We explore possible causes for liner dissociation.
\end{abstract}

\section{Introduction}

The potential for liner dissociation has been well researched and a range of push-out and lever-out strengths have been reported for different modular acetabular components. The Pinnacle acetabulum from DePuy first came into use in 2003 and although in clinical testing was found to have a lower push-out and leverout strength when compared with DePuy's older Duraloc Cup it was reported as having no greater risk of short-term dissociation [1-4].

\section{Case Report}

In April of 2005 the Orthopaedic Department began keeping electronic records of all surgeries performed in this rural hospital. Since this time 286 total hip joint arthroplasties have been performed using the Pinnacle cup. Of these; 44 have had ceramic liners, 172 have used a standard neutral polyethylene liner and 70 have used a lipped polyethylene liner. Review of these records revealed six cases of polyethylene liner dissociation. All six cases occurred with the standard neutral polyethylene Pinnacle liner and all six cases were subsequently revised using a neutral or lipped polyethylene or metal liner. In four of the six cases the femoral head size was also increased. The same surgeon who performed these six primary arthroplasties also performed three of the six revisions. All surgeries took place in the same rural hospital over a period of three years and in all cases a lateral approach for surgery was used.

\section{Case 1}

A 64 year old male underwent right total hip arthroplasty for osteoarthritis (Figure 1). A 58mm Pinnacle sector 2 acetabular cup with a neutral Marathon polyethylene liner (28mm ID x 58mm OD)

was mated via a $28 \mathrm{~mm}+5$ high offset stainless steel Articul/Eze femoral head (12/14 cone) to an uncemented No 14 Corail stem. Post-op recovery was uneventful. Post-op X-Rays showed $37^{\circ}$ of inclination of the cup (Figure 2).

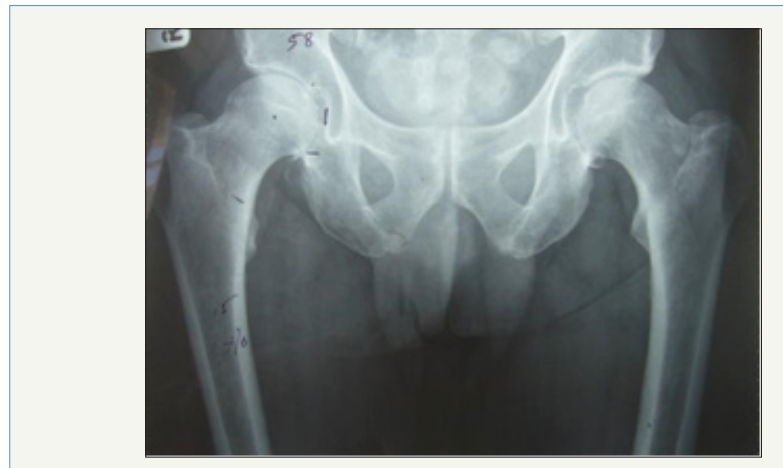

Figure 1: AP Pelvis showing right hip osteoarthritis.

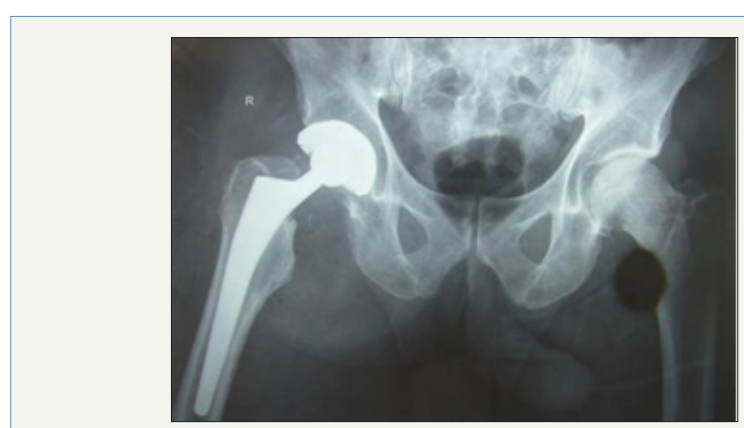

Figure 2: Post-op right total hip arthroplasty. 
At six week follow-up he did report one episode of instability a week prior when getting up from a chair and he felt a sudden "pop" in his hip with flexion but otherwise continued to mobilise independently. At 14 months post-op he presented acutely after bending forwards, again flexing at his hip, and feeling something "pop" in this hip. He was unable to mobilise. On further questioning he reported multiple episodes of instability during the past year. $\mathrm{X}$-Rays showed an eccentrically placed femoral head with no evidence of femoral or acetabular loosening or shift (Figure $3 \& 4$ )

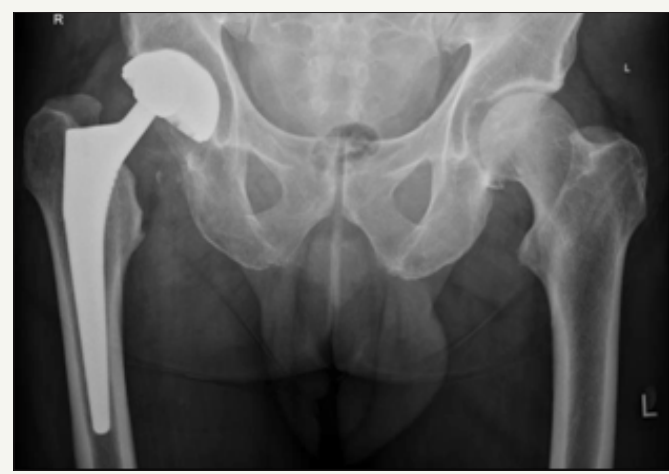

Figure 3: AP film showing disassociation.

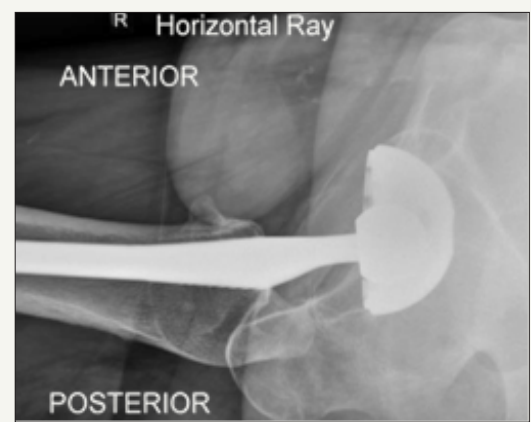

Figure 4: Liner disassociation on lateral (horizontal ray) film.

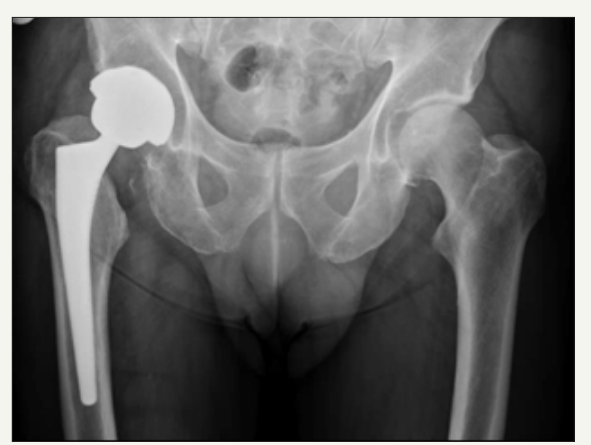

Figure 5: AP pelvis post-revision showing larger femoral head.

He underwent revision where it was found that the superior three of the six peripheral locking tabs had sheared off from the liner resulting in the liner disassociating inferiorly and anteriorly leaving the superior aspect of the cup exposed to the metal head. Cup and femoral anteversion were deemed acceptable and there was no evidence of loosening. Both were therefore retained. The disassociated liner was replaced with a neutral metal Pinnacle liner (40mm ID x 58mm OD) and the femoral head component with a $40 \mathrm{~mm}+1.5$ offset stainless steel Articul/Eze head (12/14 taper). Post-operative recovery was uneventful. At six weeks and one year post-operatively he reported no further instability (Figure 5).

\section{Case 2}

A 70 year old male underwent right total hip arthroplasty for osteoarthritis (Figure 6). A 56mm Pinnacle sector 2 acetabular cup was inserted with a neutral Marathon polyethylene liner (28mm ID x $56 \mathrm{~mm} \mathrm{OD)} \mathrm{and} \mathrm{mated} \mathrm{via} \mathrm{a} 28 \mathrm{~mm}+5$ high offset stainless steel Articul/Eze femoral head (12/14 cone) to an uncemented No 11 Corail stem. Post-op recovery was uneventful. Post-op X-Rays showed $46^{\circ}$ of inclination of the cup (Figure 7).

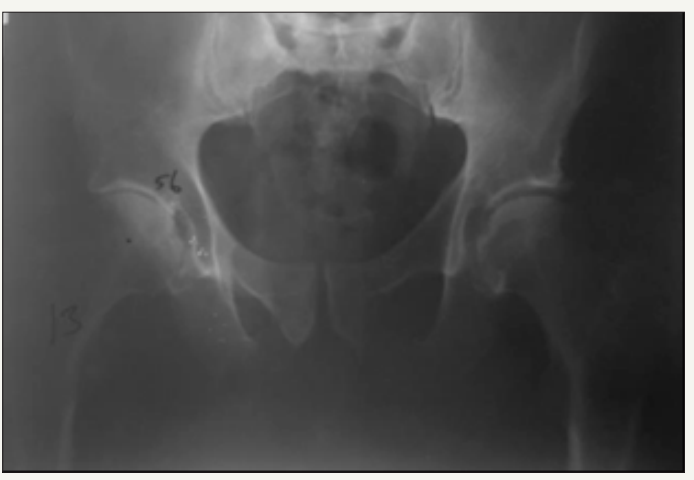

Figure 6: Pre-op AP film showing right hip osteoarthritis.

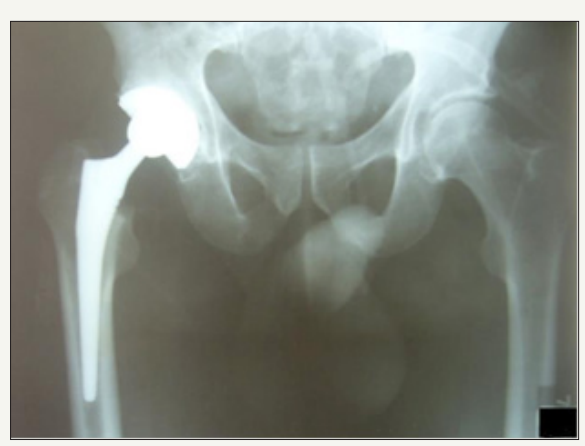

Figure 7: Post-op right total hip arthroplasty.

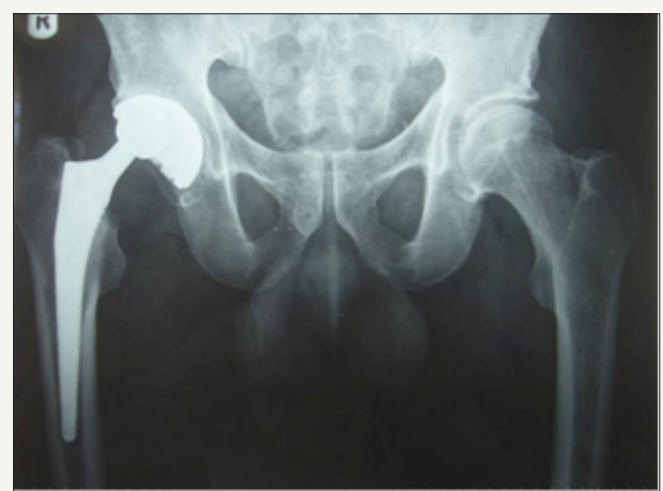

Figure 8: Right hip liner disassociation. 
Atsix week follow-up he was noted to have a slight Trendelenburg gait but was otherwise well with no reports of instability. At 19 months post-op he presented acutely after bending forwards flexing at his hip and feeling something "pop" within his hip. He was able to mobilise soon after although with some difficulty and reported feeling something "rubbing" within the hip. Again, X-Rays showed an eccentrically placed femoral head (Figure $8 \& 9$ ).

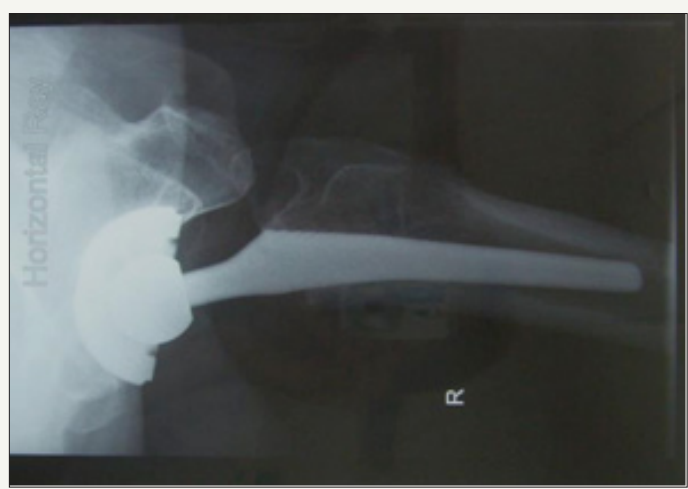

Figure 9: Lateral film showing liner disassociation.

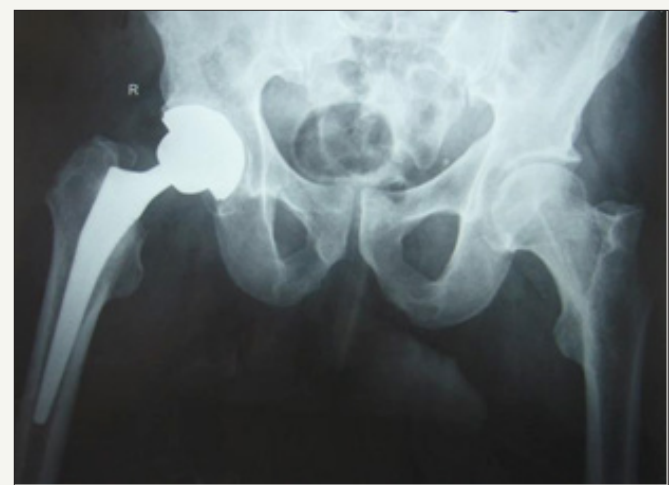

Figure 10: Post-revision film.

He underwent revision surgery where again it was found that the three consecutive superiorly placed locking tabs had sheared off resulting in the liner dissociating. Again cup and femoral anteversion were deemed acceptable and there was no evidence of loosening and both were therefore retained. The disassociated liner was replaced with a neutral metal Pinnacle liner (36mm IDx56mm OD) and the femoral head component with a $36 \mathrm{~mm}+5$ offset stainless steel Articul/Eze head (12/14 taper). Post-operative recovery was uneventful and on review in outpatient clinic 6 weeks post-op and at one year he was mobilising well with full range of movement of the hip and reported no further episodes of instability (Figure 10).

\section{Case 3}

A 67 year old female underwent right total hip arthroplasty for osteoarthritis (Figure 11). A 56mm Pinnacle sector 2 acetabular cup with a neutral Marathon polyethylene liner (28mm IDx56mm OD) was mated via a $28 \mathrm{~mm}+3$ stainless steel Elite femoral head ( $9 / 10$ taper) to a cemented No 2 high offset C-Stem (9/10 taper) femoral component. Post-op recovery was uneventful and X-Rays showed $46^{\circ}$ inclination of the cup (Figure 12).

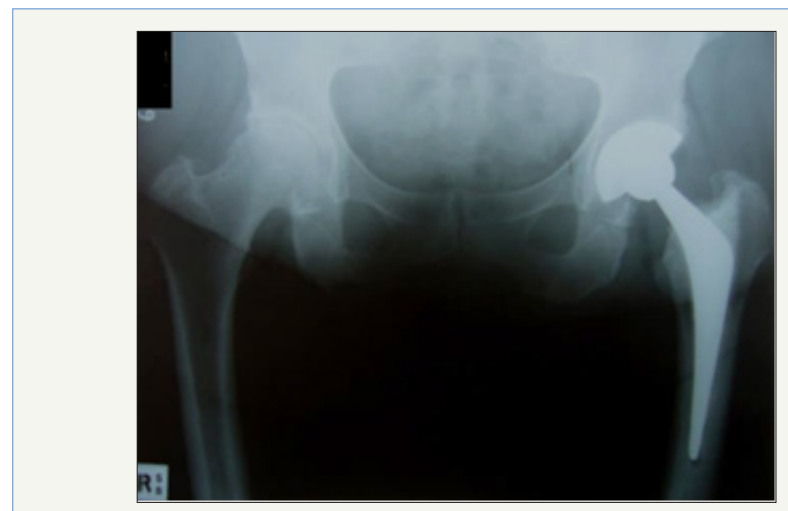

Figure 11: Pre-op film showing right hip osteoarthritis.

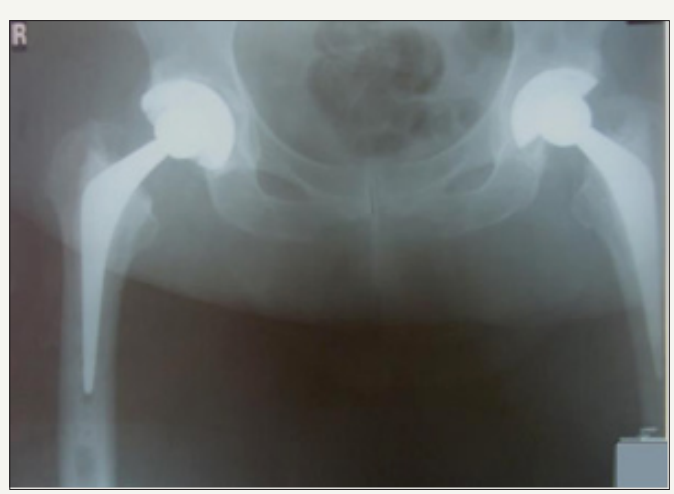

Figure 12: Post-op film showing right total hip arthroplasty.

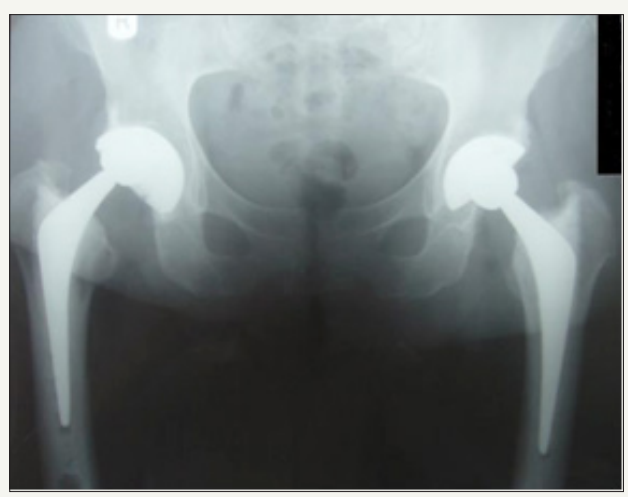

Figure 13: Right hip disassociation.

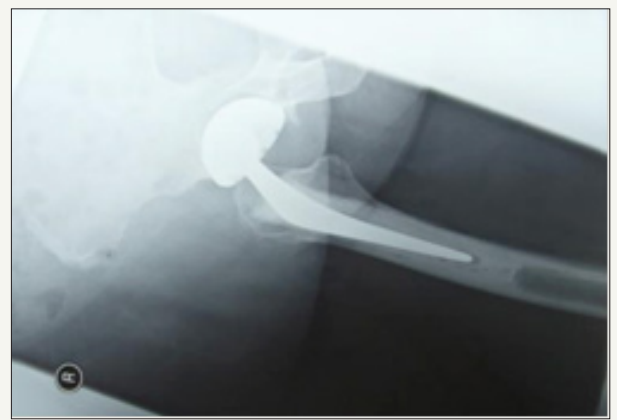

Figure 14: Lateral film of disassociation. 
At six week follow-up she was mobilising well, with good range of movement of the hip and no complaints of pain. At six months post-op she was seen acutely after feeling a pop in her hip following flexing forwards. She complained of pain in her groin and was noted to be walking with a limp. X-Rays showed an eccentrically placed femoral head (Figure 13 \& 14)

She proceeded for revision. Again the three superiorly placed locking tabs of the six had sheared off resulting in the liner lying loose. Cup and femoral anteversion were deemed acceptable and there was no evidence of loosening. The disassociated liner was replaced with a neutral metal Pinnacle liner (36mm IDx56mm OD) and the femoral head component with a $36 \mathrm{~mm}+3$ offset stainless steel Ultamet femoral head ( $9 / 10$ cone). Post-op recovery was uneventful and on review in outpatient clinic both at six weeks and two years post-op she remained well with full range of movement of the hip and no further instability (Figure 15).

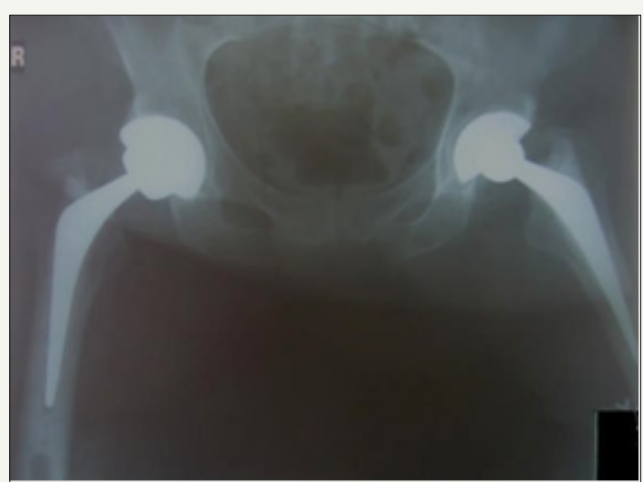

Figure 15: Post-revision.

\section{Case 4}

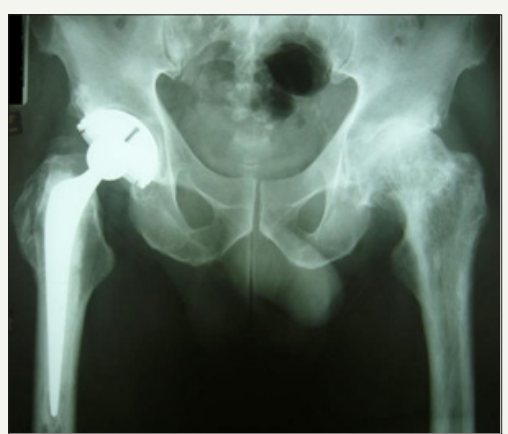

Figure 16: Pre-op showing left hip osteoarthritis.

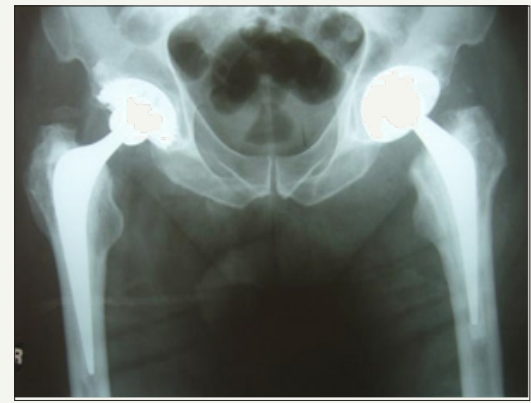

Figure 17: Post-op film showing left total hip arthroplasty.
A 73 year old male with Paget's disease underwent left total hip arthroplasty (Figure 16). A 58mm Pinnacle sector 2 acetabular cup with a neutral Marathon polyethylene liner (28mm IDx58mm OD) was mated via a $28 \mathrm{~mm}+0$ stainless steel Elite femoral head $(9 / 10$ cone) to a cemented No 3 C-Stem (9/10 taper). Post-op recovery was uneventful. Post-op X-Rays showed $40^{\circ}$ inclination of the cup (Figure 17).

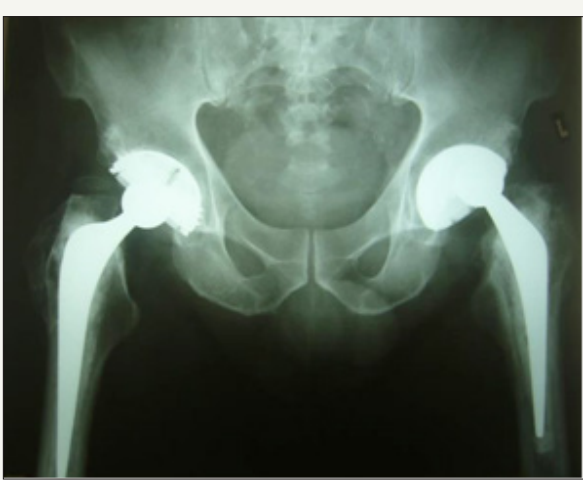

Figure 18: AP Pelvis showing eccentrically positioned femoral head within the Pinnacle acetabulum.

At six week follow-up he was mobilising well with good range of movement of the hip. At seven months post-op he saw his GP complaining of a clunking sensation in his hip and his hip feeling unstable. X-Rays were taken which showed no abnormalities. However, at 10 months post-op he was seen acutely after flexing forwards and feeling his hip "pop". X-Rays showed an eccentrically placed femoral head. Lateral films were not available (Figure 18).

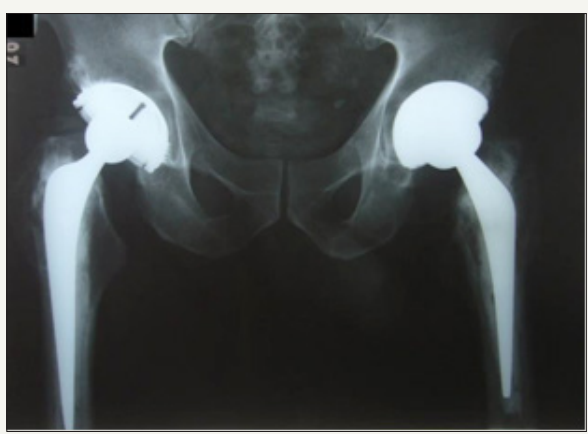

Figure 19: Post-revision film.

He proceeded for revision where it was found that the three antirotation superior locking tabs had sheared off resulting in the liner lying loose inferiorly and anteriorly. Cup and femoral anteversion were deemed acceptable and there was no evidence of loosening. The disassociated liner was replaced with a neutral Ultamet metal liner (36mm IDx56mm OD) and the femoral head component with a $36 \mathrm{~mm}+3$ offset stainless steel Ultamet femoral head ( $9 / 10$ cone). Post-op recovery was uneventful and on review in outpatient clinic at six weeks, nine months and two years he remained well with full range of movement of the hip (Figure 19).

\section{Case 5}

A 69 year old male underwent left total hip arthroplasty. He had a 56mm Pinnacle Duofix HA 100 series acetabular cup with a neutral 
Marathon polyethylene liner (28mm IDx56mm OD) implanted with a $28 m m+1.5$ stainless steel Articul/Eze femoral head (12/14 taper) and uncemented No 13 high offset Corrail femoral component. Post-op X-Rays showed $40^{\circ}$ of inclination of the cup (Figure 20). At exactly one year he presented acutely with pain in his left hip after twisting and flexing at his hip to pick up his dog. X-Rays showed an eccentrically located femoral head (Figure 21 \& 22).

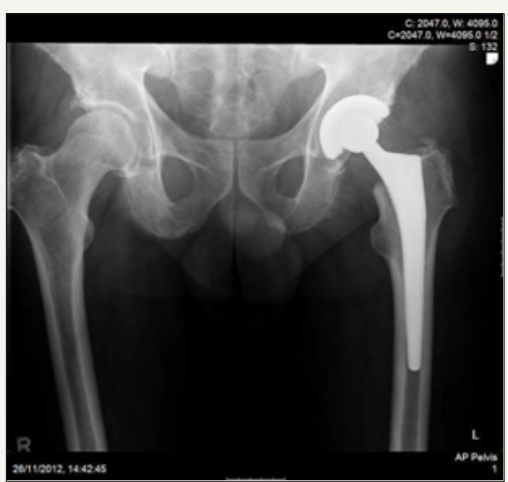

Figure 20: Post-op left total hip arthroplasty.

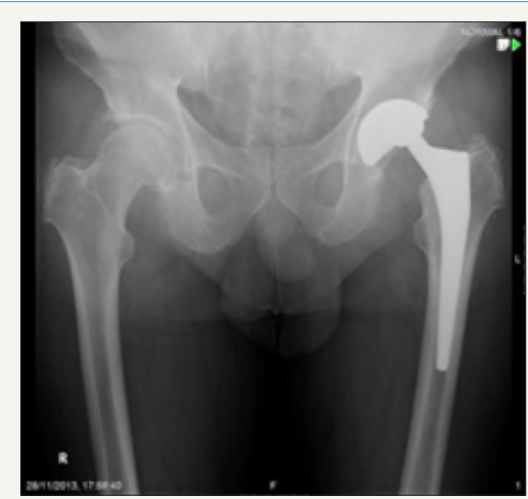

Figure 21: Dissociated liner (AP radiograph).

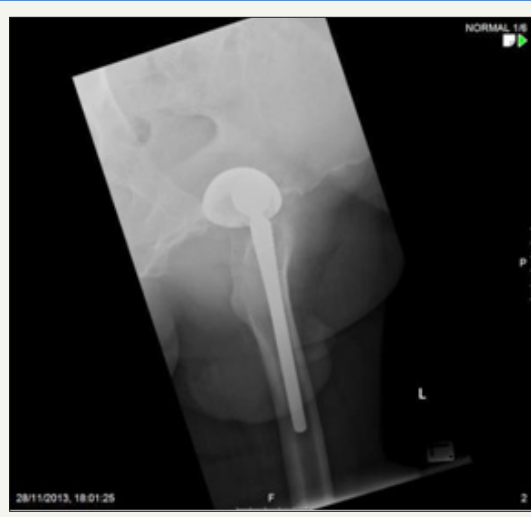

Figure 22: Dissociated liner (lateral radiograph).

He underwent revision where it was found that the 3 superior locking tabs had sheared off and the liner had spun out. The liner and head were replaced with identical sized components. At 6 weeks and one year post-op he was mobilising well and without pain and denied any further instability (Figure 23).

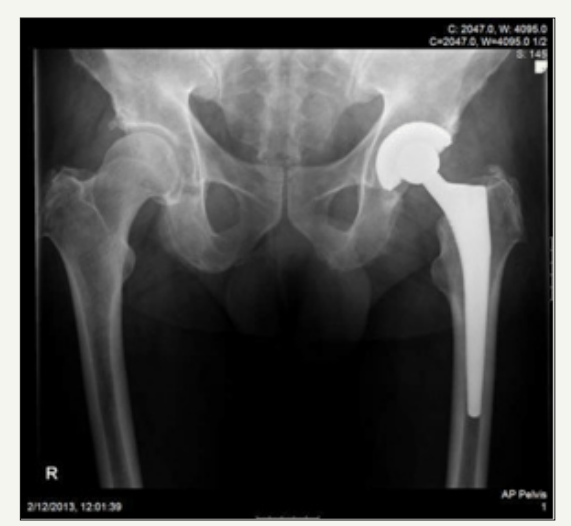

Figure 23: Post-revision surgery.

\section{Case 6}

A 58 year old male underwent left total hip arthroplasty for osteoarthritis. Implants used were a 54mm Pinnacle Duofix HA 100 series acetabular cup with a neutral Marathon polyethylene liner (28mm IDx54mm OD) implanted with a $28 \mathrm{~mm}+1.5$ Biolex Delta ceramic femoral head (12/14 taper) and uncemented No 12 lateral offset Corrail femoral component. Post-op course was uneventful with X-Rays showing $42^{\circ}$ of cup inclination (Figure $24 \& 25$ ).

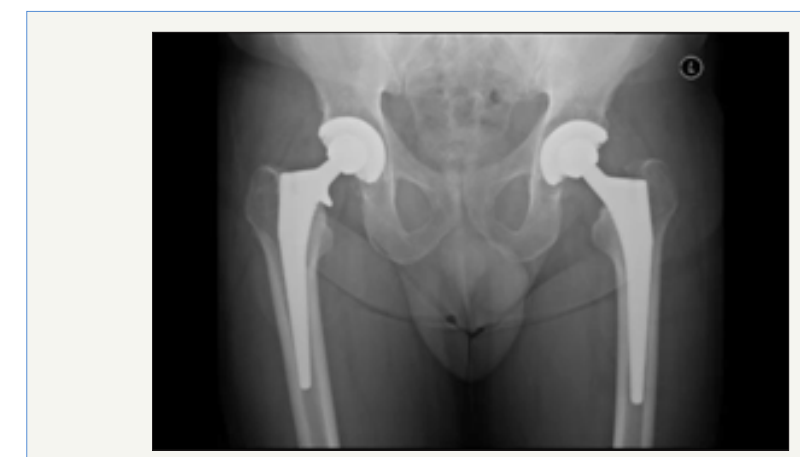

Figure 24: Post-op left total hip arthroplasty.

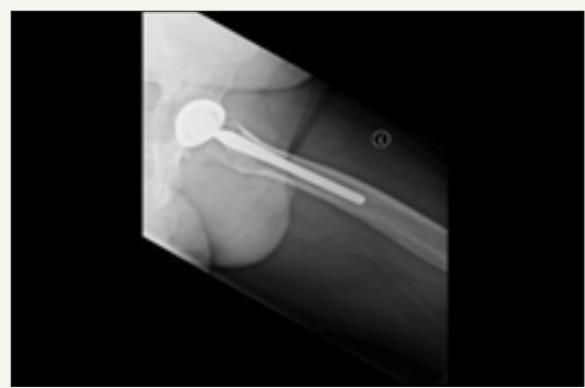

Figure 25: Post-op left total hip arthroplasty (lateral radiograph).

Four months later he was reviewed and reported four or five episodes of subluxation of the hip (whilst in the shower and when bending forwards to pick up socks). A month later he had a more severe episode in which the pain did not resolve and X-Rays showed an eccentrically positioned femoral head. He underwent revision where three of the six anti-rotation lugs were broken (although in 
this case the 3 were not adjacent lugs). The head was exchanged and a lipped polyethylene liner was placed. At 9 months post-op he continues to do well (Figure 26-29).

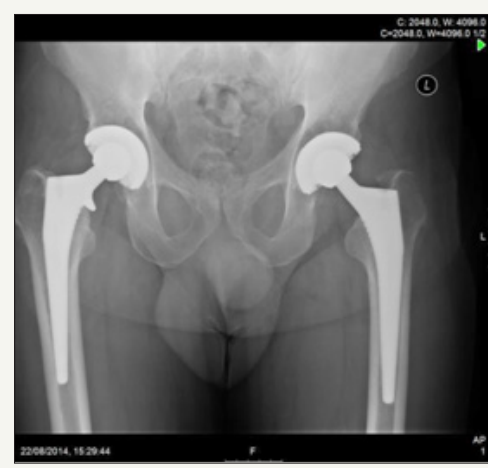

Figure 26: Post-revision left hip arthroplasty.

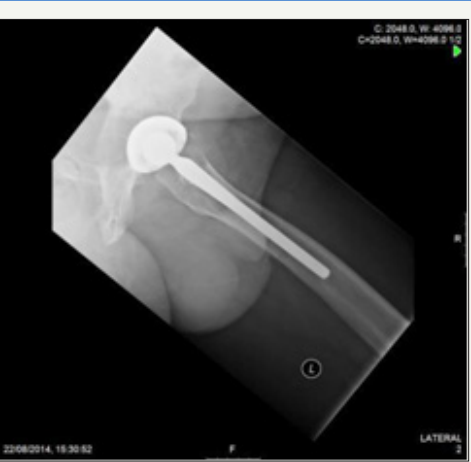

Figure 27: Post-revision left hip arthroplasty (lateral radiograph).

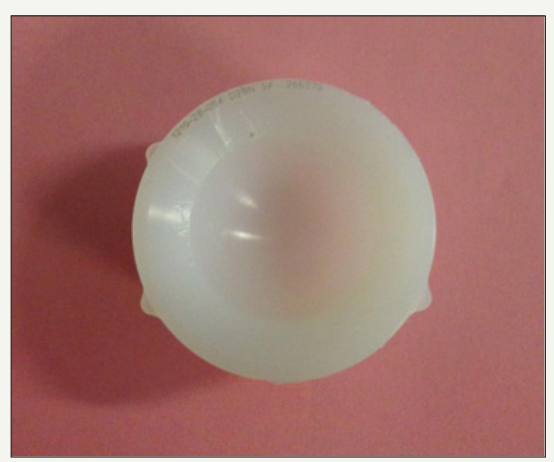

Figure 28: Dissociated Pinnacle polyethylene liner showing three of the six locking tabs have sheared off.

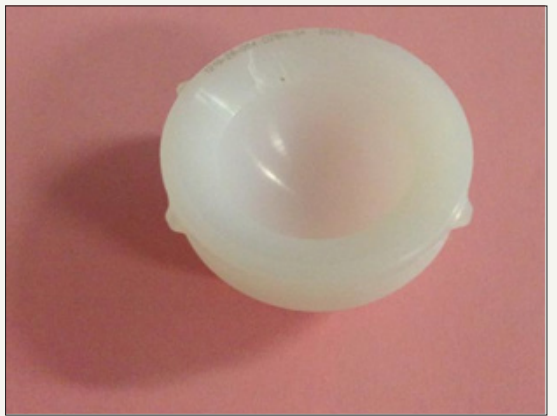

Figure 29: Only three of the six derotation locking tabs remain.

\section{Discussion}

There are similar case studies in the literature reporting liner disassociation with the Pinnacle cup. Mesko [5] reported a single case (where similarly three of the six peripheral locking tabs were found to have sheared off at revision) and also made reference to the 44 cases of Pinnacle cup polyethylene liner disassociation between June 52002 and March 172008 reported by the FDA's Manufacturer and User Facility Device Experience (MAUDE). Yun [6] reported on 23 cases of liner dissociation with the Pinnacle cup and recommended that surgeons take extra precautions when using the Pinnacle cup to ensure integrity of the locking mechanism at the time of surgery. Conversely there are studies that report no adverse outcomes. For example, Engh [4] who reported on 596 Pinnacle cups with a less than two year mean follow-up found no cases of design failure. Another multicentre study of 1183 Pinnacle cups with a two year mean follow-up found no cases of liner disassociation [7].

The cause for liner disassociation is uncertain but is likely to be multifactorial. Patient factors such as body habitus and level of activity could possibly play a role. In the cases described above all six patients were overweight. Furthermore, in all cases the patients were able to pinpoint the exact movement that resulted in the disassociation-specifically hip flexion. Whether hip flexion was excessive and enough to cause partial subluxation and liner disassociation is uncertain. In three of the six cases the patients had reported subjective episodes of instability prior to the actual definitive dissociation. Whether these episodes were partial subluxations of the liner or whether these episodes may have weakened the locking system (possibly even sheared off locking tabs) predisposing the liner to disassociation at some stage in the future cannot be concluded from this study.

Surgical factors are also likely to play a key role. All six primary total hip arthroplasties were performed through a lateral approach. Whether surgical approach is a factor is something that should be investigated further. There were 88 total hip joint arthroplasties performed in this rural hospital over the past five years since electronic record keeping began using the Pinnacle cup and a lateral approach. Of these 78 used a neutral polyethylene liner and there were four disassociations as discussed in this case report. There were 173 total hip arthroplasties performed during these five years with the Pinnacle cup and a posterior approach. There were no cases of liner disassociation in this group (91 cases used a neutral polyethylene liner).

Surgical technique is also likely to be a crucial factor and it has been postulated that imperfect seating of the liner at the time of the surgery due to interposing debris (soft tissue and bone fragments) could predispose to liner disassociation. In these four cases other than the sheared locking tabs macroscopically there were no other obvious deformities of the liners with no evidence of wear on the posterior surface suggestive of retained debris between the cup and liner being responsible for disassociation.

Malalignment of the acetabulum may result in impingement with consequent levering out the liner and thus disassociation. 
In these four cases the degree of cup inclination (which ranged from $37^{\circ}$ to $46^{\circ}$ ) was deemed appropriate. Anteversion of the cup and femoral component were also deemed appropriate both on primary arthroplasty and on revision although were not specifically measured. How important a role component alignment, or malalignment as it may be, plays in liner disassociation is also something that needs further more specific investigating.

System design is also likely to play a key role in cases of liner disassociation. The Pinnacle system design is such that the liner interlocks with the cup via an inclined taper mechanism and six peripheral locking tabs provide stability by preventing rotation. There are both non-cross-linked and cross-linked polyethylene liners. In these four case studies all liners used in the primary surgery were Marathon cross-linked liners. It has been proposed that the melting and irradiating process used to produce crosslinked liners may affect the tensile strength and consequently increase the risk of liner failure through cracks and fractures of the polyethylene [8]. Production method may therefore play a role in liner disassociation.

The liners also come in different configurations (neutral, lateralised and $10^{\circ}$ ). Of the 286 total hip joint arthroplasties that have been performed in this rural hospital using the Pinnacle cup since electronic recording keeping began 172 have used a neutral polyethylene liner. The four cases of liner disassociation discussed above occurred with these neutral liners. There were 68 cases in which a lipped polyethylene liner was used and there were no disassociations. Two cases used a $10^{\circ}$ polyethylene liner and in neither case has disassociation been reported. Therefore, whether liner configuration plays a role in disassociation and specifically whether the incidence of disassociation differs between the differently configured liners is something that should be investigated further.

Although this report has investigated only cases of polyethylene liner disassociation the particular liner used (polyethylene, metal or ceramic) may also be a factor. Anecdotally it is reported that disassociation rates are lower with metal liners (which is why the disassociated polyethylene liners were replaced with metal liners in these four cases) and this is something that should also be investigated further. These four cases of liner disassociation were all polyethylene liners and of the 44 ceramic liners that have been used in primary arthroplasties in this rural hospital since the electronic database was established five years ago there have been no reported disassociations (although there has been one case of a fractured ceramic liner).

DePuy's older Duraloc cup was found in clinical trials to have higher a push-out and lever-out strength than the Pinnacle and so whether liner disassociation is primarily a phenomenon confined to the Pinnacle system and a consequence of design failure is something that needs further investigation and perhaps a retrospective study comparing rates of liner disassociation between the Duraloc and Pinnacle would provide answers [1-4]. The Duraloc cup was superseded by the Pinnacle in this hospital and as such the electronic database has only nine cases of primary total hip arthroplasty using the Duraloc cup. In none of these cases has liner disassociation occurred.

In all four cases it was the superior three of the six peripheral locking tabs that were found to have sheared off. Whether these three tabs play more of a stabilising role than the inferiorly placed tabs or whether being positioned superiorly places the tabs under more stress thus making them more prone to fracturing is also something to ponder.

\section{Conclusion}

This article possibly raises more questions than it does answers in terms of the causal factors for liner disassociation and it is only with further investigation that more light will be shed on this relatively uncommon but extremely important complication of total hip arthroplasty with the Pinnacle cup. We suggest the need for a more vigilant reporting system for cases of liner disassociation similar to this hospital's electronic database and it is possible that with increasing numbers of cases being reported causal trends may be identified. Long-term follow-up of these patients will determine whether the decision to revise their total hip arthroplasties following polyethylene liner disassociation with larger femoral heads and metal liners is successful in preventing repeat disassociations in the future.

\section{References}

1. Tradonsky S, Postak PD, Froimson AI, Greenwald AS (1993) A comparison of the disassociation strength of modular acetabular components. Clin Orthop Relat Res 296: 154-160.

2. Retpen JB, Solgaard S (1993) Late disassembly of modular acetabular component. A report of two cases. Acta Orthop Scand 64(2):193-195.

3. Postak PD, Ratzel AR, Greenwald AS (2016) Highly crosslinked polyethylene modular acetabular designs: performance characteristics. AAOS poster, pp. 1-4.

4. Engh CA, Hopper RH, Engh CA (2004) Long term porous-coated cup survivorship suing spikes, screws and press fitting for initial fixation. Journal of Arthroplasty 19(7): 54-60.

5. Mesko JW (2009) Acute liner disassociation of a pinnacle acetabular component. Journal of Arthroplasty 24(5): 815-818.

6. Yun A, Koli EN, Moreland J, Iorio R, Tilzey JF, et al. (2016) Polyethylene liner dissociation is a complication of the DePuy Pinnacle cup: a report of 23 cases. Clin Orthop Relat Re 474(2): 441-446.

7. Kidsfater K, Barrett WF, Dowd JE (2007) Midterm survival of the Pinnacle multi-liner acetabular cup in a prospective mutli-center study. Poster P077, AAOS Annual meeting, San Diego, USA.

8. Wang A, Manley M, Serekian P (2004) Wear and structural fatigue simulation of cross-linked ultra-high molecular weight polyethylene for hip and knee bearing applications. Journal of ASTM International 1(1): 1-16. 
Creative Commons Attribution 4.0 International License

For possible submissions Click Here

Submit Article

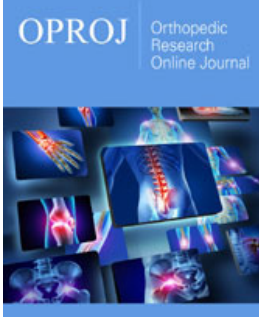

Orthopedic Research Online Journal

Benefits of Publishing with us

- High-level peer review and editorial services

- Freely accessible online immediately upon publication

- Authors retain the copyright to their work

- Licensing it under a Creative Commons license

- Visibility through different online platforms 